Purdue University Purdue e-Pubs

7-1-1987

\title{
Organic Compound Effects On Swelling And Flocculation Of Upton-Montmorillonite
}

\author{
S. Chen
}

P. F. Low

J.H. Cushman

C. B. Roth

Follow this and additional works at: http://docs.lib.purdue.edu/watertech

Chen, S.; Low, P. F.; Cushman, J. H.; and Roth, C. B., "Organic Compound Effects On Swelling And Flocculation Of UptonMontmorillonite" (1987). IWRRC Technical Reports. Paper 178.

http://docs.lib.purdue.edu/watertech/178

This document has been made available through Purdue e-Pubs, a service of the Purdue University Libraries. Please contact epubs@purdue.edu for additional information. 


\section{ORGANIC COMPOUND EFFECTS ON SWELLING AND FLOCCULATION OF UPTON - MONTMORILLONITE}

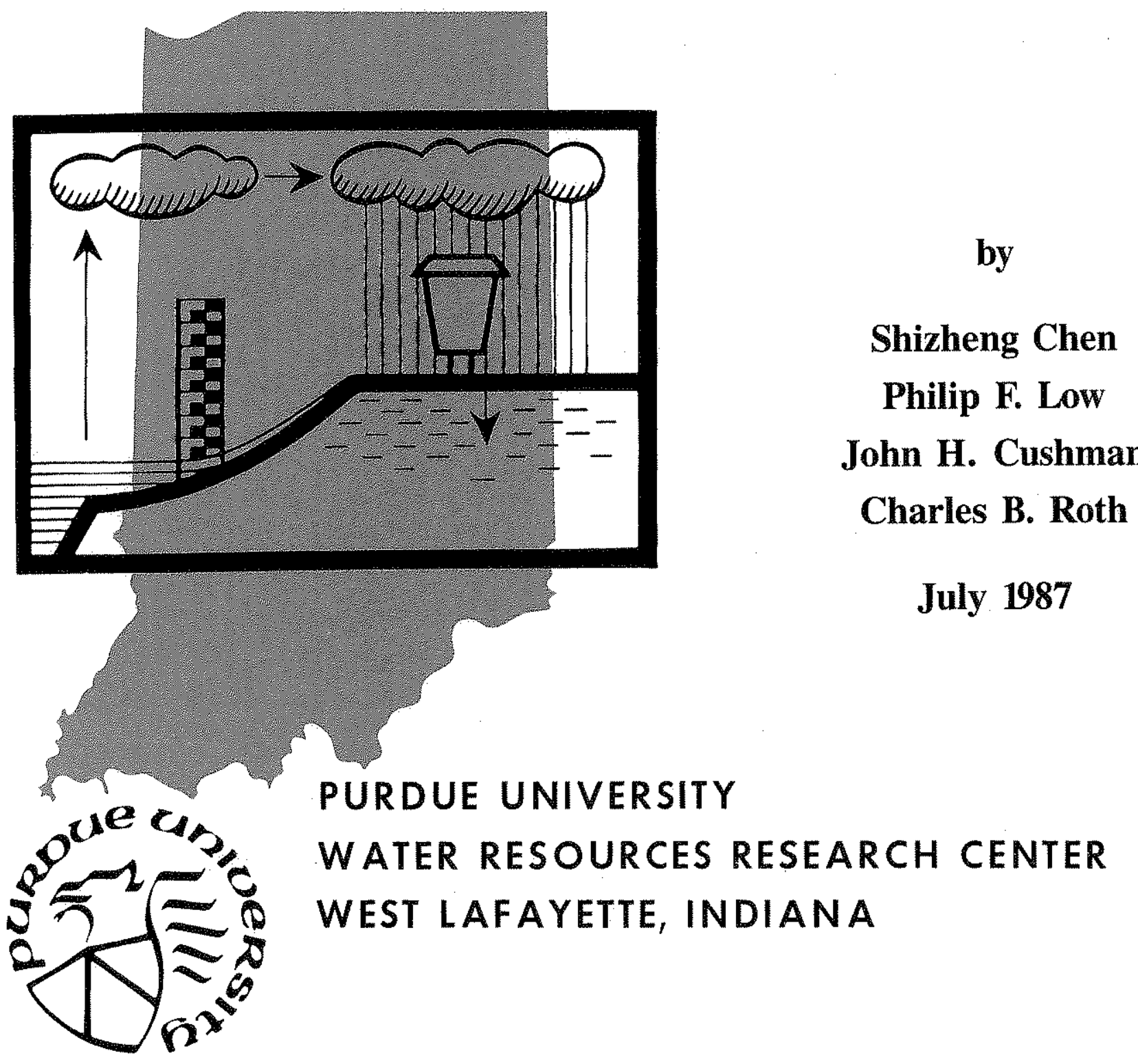


Technical Report Number 178

\title{
ORGANIC COMPOUND EFFECTS ON SWELLING AND FLOCCULATION OF UPTON-MONTMORILLONITE
}

by

Shizheng Chen

Philip F. Low

John H. Cushman

Charles B. Roth

U.S.G.S. Project Number: G1016-04

\author{
Submitted to: \\ U.S. Geological Survey \\ United States Department of the Interior \\ Reston, VA 22092
}

\footnotetext{
"The activities on which this report is based were financed in part by the Department of the Interior, U.S. Geological Survey through the Purdue University Water Resources Research Center."

"Contents of this publication do not necessarily reflect the views and policies of the United States Department of the Interior, nor does mention of trade names or commercial products constitute their endorsement by the U.S. Government."

Water Resources Research Center Purdue University, West Lafayette, IN 47907
} 
Final Technical Completion Report

Period of Investigation: June 1,1985 to June 30,1986

Project Number: G1016-04

Grant Agreement Number: 14-08-0001-G1016 


\section{ACKNOWLEDGEMENTS}

We are grateful to the Office of Health and Environmental Research of the United States Department of Energy (Grant \#DE-FG02/85ER60310) as we11 as to Purdue University and the Department of Interior for financial support of this work.

These funds allowed a visiting scholar, Mr. Shizheng Chen, to take part in the project. 
TABLE OF CONTENTS

Page

Abstract . . . . . . . . . . . . . . . . . . . . . . . . . . . . . . . . 1

Introduction . . . . . . . . . . . . . . . . . . . . . . . . . . . . . . . 2

Experimental Methods . . . . . . . . . . . . . . . . . . . . . . . 3

Results and Discussion . . . . . . . . . . . . . . . . . . . . . . . 5

References . . . . . . . . . . . . . . . . . . . . . . . . . . . 14

Figure Legend . . . . . . . . . . . . . . . . . . . . . . . . . 18 
ABSTRACT

The swelling and shrinking and flocculation and deflocculation of clays is of fundamental importance in the transport of solutes through a porous medium because these processes affect the permeability of the medium. Since organic solutes frequently occur in toxic wastes, we conducted an investigation of the effect of eight soluble organic compounds, representing different kinds of structures and functional groups, on the swelling and flocculation of sodium saturated Upton-Montmorillonite. These compounds were ethanol, 1,4-dioxane, phenol, urea, benzamide, ethylamine hydrochloride, acetic acid, and sodium acetate. In general, we found the compounds which ionize in water exerted a greater effect than those that remain unionized, i.e., electrically neutral. However, 1,4-dioxane was exceptional in that, despite its electrical neutrality, it reduced swelling and increased flocculation significantly. Possible reasons for the effects of the different compounds on swelling and flocculation were advanced. 


\section{INTRODUCTION}

A fundamental understanding of the factors affecting the rate of transport of organic solutes in porous media is of the utmost importance in controlling environmental wastes and minimizing health hazards. To determine travel times of such solutes in natural subsoils and other subsurface formations requires fundamental knowledge of clay-water-organic intexactions. Clay in a porous medium can profoundly affect the convective and diffusive transport of organic molecules through the medium. It may exert its influence in several ways, namely: (1) by swelling in place to close up the pores; (2) by disjoining from pore walls during swelling (or deflocculation), moving downstream with the flowing water and clogging the narrow throats between larger pores; (3) by interacting with the pore water to alter its properties, e.g., its viscosity and diffusivity or its ability to dissolve the organic molecules; (4) by adsorbing the organic molecules; and (5) by acting as a catalyst for organic chemical reactions.

Conversely, the influence of the clay may be modified by organic molecules. For example, these molecules may affect swelling or the degree to which the clay alters the properties of the water in contact with it (i.e., vicinal water). In other words, there are reciprocal effects. The clay affects the transport of organic molecules and the organic molecules affect the properties of the clay. Although the chemistry of clay-organic reactions has been extensively investigated (Theng, 1974) and the swelling of clay in pure oxganic solvents has been studied to a limited extent (e.g., Olejnik, et. al., 1974; Murray and Quirk, 1982; Green, et. a1., 1983), 1ittle is known about the effect of dissolved organic molecules on the behavior of the clay and on its interaction with water. The research reported in this paper was undertaken to determine how different classes of organic compounds affect clay swelling and flocculation. 


\section{EXPERTMENTAL METHODS}

Upton Montmorillonite was chosen for our experimental study because it is representative of a large class of swelling $2: 1$, silicate minerals, and because we have been studying its physico-chemical properties in the absence of organic molecules for a number of years. The Na-saturated, $<2 \mu \mathrm{m}$ Upton Montmorillonite was prepared as outlined by Low (1980). We chose eight organic compounds to study, namely: ethylamine hydrochloride, acetic acid, sodium acetate, ethanol, 1,4-dioxane, phenol, benzamide, and urea. As indicated in Figure 1, these compounds have a wide variability in structure and functional groups and they were chosen for this reason. They represent both straight-chain and ring structure and their functional groups (hydroxy, carboxy, carbonyl, amino, and oxy) either remain neutral or ionize in water. All of them have at least a small solubility in water. Two of them, 1,4-dioxane and urea, are supposed to break hydrogen bonds.

The swelling experiments were carried out as follows. The Upton clay was suspended in aqueous solutions of the different organic compounds at different concentrations. These suspensions were introduced into the miniature pressureplate apparatus described by Low (1980) and the solutions were expressed from them under an applied pressure of $0.3 \mathrm{MPa}$. Then, after the attainment of equilibrium, $\mathrm{m}_{\mathrm{w}} / \mathrm{m}_{\mathrm{c}}$, the mass ratio of water to clay in the filter cake was determined gravimetrically. It should be noted that $I$, the swelling pressure of the clay, equals the applied pressure at equilibrium.

To carry out the flocculation study, we used the following procedure. Each compound was dissolved in deionized water to make solutions of different concentration. Also, the Upton Montmorillonite was dispersed in deionized water to make a suspension containing 18 Montmorillonite by weight. A $5-\mathrm{ml}$ aliquot of this suspension was thoroughly mixed with the same volume of the different organic solutions, the $\mathrm{pH}$ was adjusted with $\mathrm{HCl}$ or $\mathrm{NaOH}$ to a specific value in some cases, 
and the mixtures were allowed to stand overnight. Then the degree of flocculation of the Montmorillonite was determined by pouring the mixture into a quartz cuvette (path length $=1 \mathrm{~cm}$ ) and measuring its absorbance, A, relative to that of a solution which was identical to the one in which the Montmorillonite was suspended. This measurement was made at two wavelengths, namely 450 and $760 \mathrm{~nm}$, by means of a double-beam, uv-visible spectrophotometer (Beckman ACTA CII). At these wavelengths, the particles should scatter the light but not absorb it (Banin and Lahav, 1968).

The turbidity, $\tau$, of a suspension of colloidal particles is a measure of the amount of light scattered by them. In keeping with the Beer-Lambert law, it is customary (e.g., Ferreiro and Helmy, 1974) to define $r$ by the expression:

$$
2.303 \log \left(\mathrm{I}_{\mathrm{o}} / \mathrm{I}\right)=r \ell=2.303 \mathrm{~A}
$$

where $I_{0}$ and $I$ are the respective intensities of the incident and transmitted light and $\ell$ is the optical path length. Further, the Rayleigh equation for light scattering may be written:

$$
r=\mathrm{kNV}^{2}
$$

where $\mathrm{k}$ is an optical constant, $\mathrm{N}$ is the number of particles per $\mathrm{cm}^{3}$, and $\mathrm{V}$ is the volume of the individual floccules. Combination of Eqs. (1) and (2) gives

$$
A=\mathrm{kNV}^{2} \ell / 2.303
$$

Equation (3) is the basis for studying flocculation by optical methods. As the volume of a floccule increases with flocculation, A increases accordingly. This is true even if the number of flocs decreases because $A$ depends on the first power of $\mathbb{N}$ but on the second power of $V$. Thus, the measurement of A for each mixture allowed us to determine the relative effects of the different organic compounds at several concentrations on the flocculation of the Montmorillonite. 
RESULTS AND DISCUSSION

Before discussing our results, it will be instructive to consider Figures 2 and 3 . Figure 2 shows the three primary ways in which planar clay particles can be arranged, namely, edge-to-edge, edge-to-face, and face-to-face. As the particles approach each other in any of these arrangements, their mutual interaction energy follows the course indicated qualitatively in Figure 3 . The mutual interaction energy is composed of contributions due to Born repulsive forces, van der Wals' attractive forces, electrostatic repulsive and attractive forces, and hydration forces, i.e., forces arising from the hydration of the particle surfaces and exchangeable cations. Consequently, the height of the energy barrier and the depth of the primary and secondary energy minima are affected by any factors that affect these forces. As the height of the energy barrier decreases, an increasing fraction of the particles has sufficient kinetic energy to surmount it and fall into the primary energy minimum. Particles in this minimum are in the flocculated state. Therefore, any factor that lowers the energy barrier will enhance the rate of flocculation; and any factor that deepens the primary energy minimum will stabilize the flocculated state.

Swelling is the moving apart or disjoining of clay particles, especially those in the parallel arrangement, until they reach their equilibrium separation under the given pressure or until they fall into the secondary energy minimum. In the process, water is absorbed and the clay matrix expands but remains intact. Deflocculation is the moving apart or disjoining of clay particles in any arrangement until they become completely dispersed in the surxounding solution. Shrinking is the reverse of swelling and flocculation is the reverse of deflocculation. Hence, swelling and shrinking are similar to, and involve the same kinds of forces, as deflocculation and flocculation, respectively. 
The diameters of the pores in a porous medium are reduced when the clay in the medium swells in place or the deflocculated particles are swept downstream in the flowing water and lodge in the narrow throats between the larger pores. On the other hand, the diameters of the pores are increased when the clay in the medium shrinks or the particles are flocculated in place. According to Poiseuille's law, the rate of flow through a pore is proportional to the second power of its diameter. Therefore, the swelling and flocculation of the clay in a porous medium must affect the permeability of the medium and, thereby, the convective transport of dissolved solutes through it.

Attention is called to the fact that a clay crystal consists of a stack of essentially parallel clay layers that are separable by water and that, within such a stack, more than one interlayer distance can exist (e.g., Rhoades, et. al., 1969; Viani, et. al., 1983, 1985). Usually, however, there is one relatively large interlayer distance, $\lambda_{e}$, that varies with the swelling pressure, $\Pi$, and relatively small interlayer distance, $\lambda_{\mathrm{p}}$, that is independent of $I \mathrm{l}$ and remains fixed at $\approx 1.0 \mathrm{~nm}$. As the fraction $f_{e}$, of layers separated by $g_{e}$ increases, the fraction, $f_{p}$, of layers separated by $\lambda_{p}$ necessarily decreases because $f_{e}+f_{p}=1$. The volume of an aggregate depends on the volume of the individual crystals, the number of crystals composing the aggregate and the arrangement of crystals within it. Note that the volume of an individual crystal depends on $f_{e}, \lambda_{e}$, and the number of layers it contains.

In condensed clay gels, the distribution of the water between the intercrystal and interlayer (intracrystal) regions depends somewhat on $\lambda_{e}$ and $f_{e}$ but mostly on the arrangement of the crystals with respect to each other. As this arrangement becomes more random, the intercrystal regions increase in volume relative to the interlayer regions, and $\gamma$, the fraction of water in the interlayer regions, decreases. 
In view of the above discussion,

$$
\gamma \mathrm{m}_{w} / \mathrm{m}_{\mathrm{c}}=\frac{\mathrm{s}}{2}\left[\mathrm{f}_{\mathrm{e}} \int_{0}^{\lambda_{\mathrm{e}}} \rho_{\mathrm{e}} \mathrm{dz}+\hat{f}_{\mathrm{p}} \int_{0}^{\lambda} \rho_{\mathrm{p}} \mathrm{dz}\right]
$$

and, hence,

$$
\begin{aligned}
\frac{\mathrm{m}_{\mathrm{w}}}{\mathrm{m}_{\mathrm{c}}} & =\frac{1}{2 \gamma} \mathrm{Sf}_{\mathrm{e}}\left(\int_{0}^{\lambda_{\mathrm{e}}} \rho_{\mathrm{e}} \mathrm{dz}-\int_{0}^{\lambda_{\mathrm{p}}} \rho_{\mathrm{p}} \mathrm{dz}\right)+\frac{1}{2 \gamma} \mathrm{s} \int_{0}^{\lambda_{\mathrm{p}}} \rho_{\mathrm{p}} \mathrm{dz} \\
& =\frac{1}{2 \gamma} \mathrm{Sf}_{\mathrm{e}}\left(\lambda_{\mathrm{e}} \bar{\rho}_{\mathrm{e}}-\lambda_{\mathrm{p}} \bar{\rho}_{\mathrm{p}}\right)+\frac{1}{2 \gamma} \mathrm{s} \lambda_{\mathrm{p}} \bar{\rho}_{\mathrm{p}}
\end{aligned}
$$

where $S$ is the specific surface area of the clay, $\rho_{p}$ and $\rho_{e}$ are the densities of water anywhere between the expanded and partially expanded layers respectively, $z$ is a coordinate taken normal to the surface, and $\mathrm{m}_{\mathrm{w}} / \mathrm{m}_{\mathrm{c}}$ is the mass ratio of water to clay. Equation 5 shows that, for any given clay at a specific value of II, $\mathrm{m}_{\mathrm{w}} / \mathrm{m}_{\mathrm{c}}$ will vary with $\mathrm{f}_{\mathrm{e}}, \lambda_{e}$, and $\gamma$ as they are affected by organic molecules in the solution.

In extensive studies of the swelling of Upton Montmorillonite and other smectites, Low and his coworkers (Low, 1980, 1981, 1987; Viani, et. al., 1983, 1985) have established the fact that the swelling of clays is due primarily to the hydration of their layer surfaces and that double-layer repulsion plays only a negligible role. The reader should also consult Schoen, et. al. (1987) for a theoretical discussion of the enormous pressures generated by surface hydration of a neutral raxe gas fluid in a slit pore. Therefore, if dissolved organic molecules affect the swelling of clay as indicated by $\mathrm{m}_{\mathrm{w}} / \mathrm{m}_{\mathrm{c}}$, it must be because they interfere with surface hydration and affect either $f_{e}$ or $\lambda_{e}$ at the given value of $\pi$. 
Although the reader should consult the references cited above for evidence that surface hydration plays the primary role in clay swelling, it should be noted here that the effect of electrolytes on swelling is not necessarily due to their repression of electric double layers. The ions of a dissolved electrolyte interact with water molecules, and thereby, influence their arrangement. Hence, they would be expected to affect the integrity and extent of the hydration shells surrounding the clay particles. The overlap of these hydration shells is supposed to be responsible for the development of the swelling pressure. In this regard, attention is called to the fact that unionized solutes would not be expected to affect the electric double layers, except as they influence the dielectric constant, but they would be expected to affect the hydration shells to a degree depending on their interaction with the water molecules. In a later paragraph, the role of the dielectric constant will be discussed.

Figure 4 illustrates the swelling results that we obtained. In general, as the concentration of the organic compound increases, the value of $\mathrm{m}_{\mathrm{w}} / \mathrm{m}_{\mathrm{c}}$ decreases, i.e. swelling decreases. However, different organic compounds affect $\mathrm{m}_{\mathrm{W}} / \mathrm{m}_{\mathrm{c}}$ differently. Those compounds that ionize in water (ethylamine hydrochloride, sodium acetate, acetic acid, and to a smaller extent, benzamide) tend to have the greatest effect, whereas, with the exception of dioxane, the electrically neutral compounds (ethyl alcohol, phenol, and urea) tend to have the least effect. Note that the data for benzamide axe limited because of its low solubility.

On ionizing, both the ethylamine hydrochloride and benzamide yield organic cations. These cations exchange with the adsorbed $\mathrm{Na}^{+}$(Cowan and White, 1958; Farmer and Mortland, 1965). Hydrogen bonds may also be formed between the $\mathrm{NH}_{2}$ groups and surface oxygens. Thus, both cations are held to the surface with their hydrophobic (non-polar) groups pointing outwards. The net result is that they are concentrated where they can exert the greatest disruptive effect on surface 
hydration. It is also possible that water is expelled from between adjacent clay layers because a lower energy state is achieved when the hydrophobic groups and water are separated. In other words, it is also possible that hydrophobic bonds are formed between proximate hydrophobic groups extending from opposite layer surfaces. Although there is undoubtedly a difference in the interaction of the two organic cations with the water because of their difference in structure, the greater effect of the ethylammonium ion is probably due largely to its higher concentration. Recall that ethylamine hydrochloride is more highly ionized than benzamide at any given molality. Theng, et. al., (1968) have also observed that ethylammonium ions reduce the swelling of Montmorillonite.

Both acetic acid and sodium acetate yield organic anions. These anions are repelled electrostatically from the negatively charged clay surfaces. Although acetic acid may form hydrogen bonds with the oxygens that compose these surfaces (Kohl and Taylor, 1961), it would likely do so only in its unionized state and the resulting adsorption would be small. This would be the case especially from the solution of sodium acetate, which is highly ionized. Note, however, that the sodium acetate has a greater effect on swelling than the acetic acid. It is reasonable to conclude, therefore, that $\mathrm{Nat}$ and $\mathrm{H}+$ are the components of the respective electrolytes that interfere with surface hydration and reduce swelling.

Ethanol, phenol, urea, and dioxane are electrically neutral. It has been shown (e.g., Larson and Sherman, 1964; Mortland, 1966; Bissada, et. a1., 1967; Dowdy and Mortland, 1968; Brindley, et. al., 1969; Farmer and Ahlxichs, 1969) that such compounds, when they are in the pure state or highly concentrated, are capable of forming different kinds of bonds with the clay or its exchangeable cations. However, it does not necessarily follow that these bonds are stronger than clay-water bonds and, hence, will cause the compounds to be appreciably adsorbed in the presence of an excess of water; nor does it necessarily follow 
that the compounds, if adsorbed, will disrupt the hydration shells surrounding the clay layers. Nevertheless, it should be noted that ethanol is adsorbed from dilute aqueous solutions by Na-Montmorillonite (German and Harding, 1969).

From Figure 4 it appears that ethanol and phenol had essentially no influence on swelling and that the influence of urea was slight. On the other hand, the influence of dioxane was highly significant, being comparable to that of acetic acid. In keeping with these results, Alfrey and Rodewald (1949) found that alcohol had no effect but dioxane had a profound effect on the gelation of bentonite. The structure of water is supposed to be disrupted by both dioxane (Hovorka, et. al., 1936; Oster, 1946; Alfrey and Rodewald, 1949) and urea (Rupley, 1964; Walrafen, 1966; Franks and Clarke, 1967; Hammes and Schirme1, 1967; Frank and Franks, 1968); but the structure-breaking effect of urea has been questioned (Abu-Hamdiyyah, 1965; Bonner and Woolsey, 1968; Subramanian, et. al., 1969). Consequently, it may be presumed that the dioxane and, possibly, the urea disrupt the water in the hydration shells of the layers and, in this way, cause a reduction in swelling. The question that remains, however, is why the magnitudes of their effects are so different. This question warrants further investigation. As mentioned earlier, unionized solutes such as ethanol and dioxane would not be expected to influence the electrical double layers of the clay particles unless they influence the dielectric constant of the solution. Harned and Owen (1943) reported the dielectric constants of aqueous solutions of organic solvents at different percentages of water. Included were aqueous solutions of ethanol and dioxane. If their data are used to plot the dielectric constants of the solutions of ethanol and dioxane against the molar concentrations of the respective solvents, straight lines are obtained up to a concentration of at least $1.0 \mathrm{M}$. From these lines, we find that the rate of decrease of the dielectric constant with increasing solute concentration is $2.5 / \mathrm{M}$ with ethanol and $6.7 / \mathrm{M}$ with dioxane, 
i.e., the rate of decrease with ethanol is $37 \%$ of that with dioxane. Yet, ethanol had no significant effect on swelling and dioxane had a marked effect. Moreover, at a dioxane concentration of $1.0 \mathrm{M}$, the loss in dielectric constant was -8.58 but the loss in swelling (as indicated by $\mathrm{m}_{\mathrm{w}} / \mathrm{m}_{\mathrm{C}}$ ) was $\sim 50 \%$. In other words, dioxane had a far greater effect on swelling than it did on the dielectric constant. Therefore, it is reasonable to conclude that the effects of organic solutes on swelling cannot be ascribed to their effects on the dielectric constant.

Illustrated in Figure 5 and 6 are the major results of our experiments on flocculation. These results do not allow us to specify the arrangement of the particles in the floccules but they do allow us to conclude that, excepting dioxane, the neutral organic molecules (ethanol, phenol, and urea) have little or no effect on flocculation, as indicated by the magnitude of $A$, and the electrolytes (ethylamine hydrochloride, acetic acid, sodium acetate, and benzamide) have a significant effect. Dioxane also has a significant effect.

The results in Figure 5 and 6 were obtained without regard to the $\mathrm{pH}$ of the suspension. However, to examine the effect of $\mathrm{pH}$ on flocculation, the absorbance of each suspension was measured at different $\mathrm{pH}$ values in the range from 5 to 8 . The results with ethanol as solute are shown in Fig. 7. Similar results were obtained with the other organic solutes. These results show that pH had no effect. They also show that a positive edge charge was not involved in the flocculation that occurred because such a charge would be very $\mathrm{pH}$-dependent (Schofield and Samson, 1954; Rand and Melton, 1977). A similar conclusion applies with respect to the role of a positive edge charge in the rheological behavior of Montmorillonite (Rand, et. a1., 1980).

Relations between swelling and flocculation as affected by the different organic solutes are presented in Figure 8 to 10 . To properly interpret these 
figures, recall that $m_{w} / m_{c}$ increases with swelling and that $A$ increases with flocculation. Although the figures show that swelling and flocculation are inversely related, as expected, they also show that the effect of an organic solute on swelling is not always commensurate with its effect on flocculation, and vice versa. For example, urea and ethanol have a small but significant effect on $\mathrm{m}_{\mathrm{W}} / \mathrm{m}_{\mathrm{c}}$ but essentially no effect on $\mathrm{A}$. These observations suggest that, when urea and ethanol are present, the energy barrier in the curve of interaction energy vs. distance (Figure 3) is quite high so that, as this curve shifts downwards with increasing solute concentration, the interparticle distance decreases at the given II but the energy barrier is still too high to allow many particles to enter the primary energy minimum and establish equilibrium between the flocculated state (at the bottom of the primary energy minimum) and the deflocculated state (to the right of the energy barrier). Thus, swelling decreases but flocculation remains relatively unaffected.

We previously noted that the swelling of clay is due primarily to the hydration of the planar surfaces of those particles that are in the face-to-face arrangement. Hence, we interpreted the effects of the various organic compounds in terms of their effects on surface hydration. It is possible, however, that surface hydration does not influence flocculation like it does swelling. Flocculation may depend more on edge-to-face and edge-to-edge arrangements and the relative importance of surface hydration and double-layer repulsion may be different on the edges of the particles than on their planar surfaces. Consequently, to explain our results, we will recognize that both hydration and double-layer forces may be operative at the edges of particles and offer the following proposals: (1) ethylamine hydrochloride, benzamide, acetic acid and sodium acetate enhance flocculation because they are electrolytes and yield ions that interfere with surface hydration and repress the electric double layer; (2) 
the organic cations produced by the ethylamine hydrochloride and benzamide interfere with surface hydration and repress the electric double layer more effectively than inorganic cations because their non-polar groups make them less compatible with the surrounding water and strengthen their adsorption onto the surface by forming additional bonds (e.g., hydrogen bonds) with it; (3) acetate anions produced by acetic acid and sodium acetate have little effect on flocculation because they are repelled from the negatively charged surfaces; (4) ethylamine hydrochloride and sodium acetate are more effective flocculants than benzamide and acetic acid, respectively, because they are more highly ionized; (5) ethyl alcohol, phenol, and urea have little effect on flocculation because they are not adsorbed onto the surface to any significant extent, and being non-ionic, have little influence on the electric double layer; and (6) dioxane is an effective flocculant because it breaks down the structure of water and, therefore, interferes with surface hydration. Attention is called to the fact that, if the latter proposal is valid, surface hydration must be an important factor in controlling flocculation. However, regardless of the validity of any of these proposals, it is safe to conclude that, depending on their functional groups, structure and concentration, organic molecules are capable of causing Montmorillonite to flocculate or swell to different degrees and thus affect the medium permeability to different degrees. 


\section{References}

Abu-Hamdiyyah, M., 1965. The Effect of Urea on the Structure of Water and Hydrophobic Bonding. J. Phys. Chem. 69:2720-2725.

Alfrey, T., and Rodewald, C.W., 1949. An Experimental Study of Thixotropy in Bentonite Suspensions. J. Colloid Sci. 4:283-298.

Banin, A., and N. Lahav. 1968. Particle size and optical properties of montmorillonite in suspension. Israel $\mathrm{J}$. Chem. 6: 235-250.

Bissada, K.K., Johns, W.D., and Cheng, F.S. 1967. Cation-Dipole Interactions in Clay-Organic Complexes. Clay Minerals 7:155-166.

Bonner, O.D., and Woolsey, G.B., 1968. The Effect of Solutes and Temperature on the Structure of Water. J. Phys. Chem. 72:899-905.

Brindley, G.W., Wiewiora, K., and Wiewiora, A., 1969. Intracrystalline Swelling of Montmorillonite in Some Water-Organic Mixtures (Clay-Organic Studies, XVII). Amer. Mineral. 54:1635-1644.

Cowan, C.T., and White, D., 1958. The Mechanism of Exchange Between Sodium Montmorillonite and Various N-Primary Aliphatic Amine Salts. Trans. Faraday Soc. $54: 691-697$.

Dowdy, R.H., and Mortland, M.M., 1968. Alcohol-Water Interactions on Montmorillonite Surfaces: II Ethylene Glycol. Soil Sci.105:36-43.

Farmer, W.J. and Ahlrichs, J.L., 1969. Infrared Studies of the Mechanism of Adsorption of Urea-d4, Methylurea-d3, and 1,1-Dimethylurea-d2 by Montmorillonite. Soil Sci. Soc. Am. Proc. 33:254-258.

Farmer, V.C. and Mortland, M.M., 1965. An Infrared Study of Complexes of Ethylamine with Ethylamonium and Copper Ions in Montmorillonite. J. Phys. Chem. $69: 683-687$.

Ferreiro, E.A., and A.K. Helmy. 1974. Flocculation of sodium montmorillonite by electrolytes. Clay Minerals 10: 203-213. 
Frank, H.S. and Franks, F., 1968. Structural Approach to the Solvent Power of Water for Hydrocarbons; Urea as a Structure Breaker. J. Chem. Phys. 48: 4746 4757.

Franks, F., and Clarke, D.L., 1967. Solubilities of Alkylammonium Iodides in Water and Aqueous Urea. J. Phys. Chem. 71: 1155-1156.

German, W.L. and Harding, D.A., 1969. The Adsorption of Aliphatic Alcohols by Montmoxillonite and Kaolinite. Clay Minerals 8:213-227.

Green, W.J., Lee, G.F., Jones, R.A., and Palit, R. 1983. Interaction of Clay Soils with Water and Organic Solvents: Implications for the Disposal of Hazardous Wastes. Env. Sci. and Tech. 17:278.

Hammes, G.G., and Schimmel, R.P., 1967. An Investigation of Water-Urea and WaterUrea-Polyethylene Glycol Interactions. J. Am. Chem. Soc. 89:442-446.

Harned, H.S. and Owen, B.B., 1943. The Physical Chemistry of Electrolytic Solutions. Reinhold Publishing Corp., New York, NY, 611 pgs.

Hovorka, F., Schaefer, R.A., and Dreisbach, D., 1936. The System Dioxane and Water. J. Am. Chem. Soc. 58:2264-2267.

Koh1, R.A. and Taylor, S.A., 1961. Hydrogen Bonding Between the Carbonyl Group and Wyoming Bentonite. Soil Sci, 91:223-227.

Larsen, G.D. and Shexman, L.R., 1964. Infrared Spectrophotometric Analyses of Some Carbonyl Compounds Adsorbed on Bentonite Clay. Soil Sci. 98:328-331.

Low, P.F., 1980. The Swelling of Clay: II. Montmorillionites. Soil Sci. Soc. Am. J. 44:667-676.

Low, P.F., 1981. The Swelling of Clay. III. Dissociation of Exchangeable Cations. Soil Sci. Soc. Amer. J. 45:1074-1078. Low, P.F., 1987. Structural Component of the Swelling Pressure of Clays.
Langmuir, Vol. 3, No. 1 .

Mortland, M.M., 1966. Urea complexes with Montmorillonite: An Infrared Absorption Study. Clay Minerals 6:143-156. 
Murray, R.S., and Quirk, J.P., 1982. The Physical Swelling of Clays in Solvents. Soil. Sci. Soc. Amer. J. 46: 865-868.

Olejnik, S., Posner, A.M., and Quirk, J.P., 1974. Swelling of Montmorillonite in Polar Organic Liquids. Clays Clay Minerals 22:361-365.

Oster, G., 1946. The Dielectric Properties of Liquid Mixtures. J. Am. Chem. Soc. $68: 2036-2041$.

Rand, B. and I.E. Melton. 1977. Particle interactions in aqueous kaolinite suspensions. 1. Effect of $\mathrm{pH}$ and electrolyte upon the mode of particle interaction in homoionic sodium kaolinite suspensions. J. Colloid Interface Sci. $60: 308-320$.

Rand, B., E. Pekenc, J.W. Goodwin, and R.W. Smith. 1980. Investigation into the existence of edge-face coagulated structures in Na-montmorillonite suspension. J . Chem. Soc. Faraday Trans. 76: 225-235.

Rhoades, J.D., Ingvalson, R.D., and Stumpf, H.T., 1969. Interlayer Spacings of Expanded Clay Minerals at Various Swelling Pressures: An X-Ray Diffraction Technique for Direct Determination. Soil Sci. Soc. Amer. Proc. 33:473-475.

Rupley, J.A., 1964. The Effect of Urea and Amides Upon Water Structure. J. Phys. Chem. 68:2002-2003.

Schoen, M., D.J. Diestler, and J.H. Cushman, 1987, Fluids in micropores. I: Structure of a Simple Classical Fluid in a Slit Pore, J. Chem. Phys. (in press).

Schofield, R.K., and H.R. Samson. 1954. Flocculation of kaolinite due to the attraction of oppositely charged crystal faces. Disc. Faraday Soc. 18: 135-145.

Subramanian, S., Balasubramanian, D., and Ahluwalin, J.C., 1969. Nuclear Magnetic Resonance and Thermochemical Studies on the Influence of Urea on Water Structure. J. Phys. Chem. 73:266-274.

Theng, B.K.G., Greenland, D.J., and Quirk, J.P., 1968. The Effect of Exchangeable Alkylammonium Ions on the Swelling of Montmorillonite in Water. Clay Minerals $7: 271-293$.

Theng, B.K.G., 1974. The Chemistry of Clay-Organic Reactions. Adam Hilger, Ltd. 
Viani, B.E., Low, P.F., and Roth, C.B., 1983. Direct Measurement of the Relation Between Interlayer Force and Interlayer Distance in the Swelling of

Montmorillonite. J. Colloid Interface Sci., 96:229-244.

Viani, B.E., Roth, C.B., and Low, P.F., 1985. Direct Measurement of the Relation Between Swelling Pressure and Interlayer Distance in Li-Vermiculite. Clays and Clay Minerals 33:244-250.

Walrafen, G.E., 1966. Raman Spectral Studies of the Effects of Urea and Sucrose on Water Structure. J. Chem. Phys. 44:3726-3727. 
Figure Legend

Figure 1: The structure of the eight organic compounds used in this study.

Figure 2. Primary arrangement of clay particles: (a) edge-to-edge, (b) edge-toface, and (c) face-to-face

Figure 3. Diagram of interaction energy vs, interparticle distance for any particle arrangement.

Figure 4: The effect of the concentration of the organic compounds on $\mathrm{m}_{\mathrm{w}} / \mathrm{m}_{\mathrm{c}}$, the mass ration of water to clay, at a pressure Il $=0.3 \mathrm{M} \mathrm{Pa}$.

Figure 5. Absorbance, $\mathrm{A}$, of the $\mathrm{Na}$-montmorillonite suspension as a function of the molar concentration of different organic solutes at a wavelength of $450 \mathrm{~nm}$.

Figure 6. Absorbance, $\mathrm{A}$, of the Na-montmorillonite suspension as a function of the molar concentration of different organic solutes at a wavelength of $760 \mathrm{~nm}$.

Figure 7. Absorbance, A, of the montmorillonite suspension as a function of $\mathrm{pH}$ at different molar concentrations of ethanol.

Figure 8. Relation between swelling and flocculation as indicated by $\mathrm{m}_{\mathrm{w}} / \mathrm{m}_{\mathrm{C}}$ and $A$, respectively, for $\mathrm{Na}$-montmorillonite in aqueous solutions of benzamide, ethylamine hydrochloride, and urea (Note: molar concentrations are in brackets).

Figure 9. Relation between swelling and flocculation, as indicated by $\mathrm{m}_{\mathrm{w}} / \mathrm{m}_{\mathrm{c}}$ and A, respectively, for $\mathrm{Na}$-montmorillonite in aqueous solutions of phenol, 1,4-dioxane, and ethanol (Note: molar concentrations are in brackets).

Figure 10. Relation between swelling and flocculation, as indicated by $\mathrm{m}_{\mathrm{w}} / \mathrm{m}_{\mathrm{c}}$ and A, respectively, for Na-montmorillonite in aqueous solutions of sodium acetate and acetic acid (Note: molar concentrations are in brackets). 


\section{Figure 1}

The structure of organic compounds

Ethanol

$$
\mathrm{CH}_{3} \mathrm{CH}_{2} \mathrm{OH}
$$<smiles>CCO</smiles>

1,4-Dioxane

$\mathrm{C}_{4} \mathrm{H}_{8} \mathrm{O}_{2}$<smiles>C1CCOC1</smiles>

Phenol

$\mathrm{CH}_{6} \mathrm{H}_{5} \mathrm{OH}$<smiles>OC1[C]CCCC1</smiles>

Urea

$\mathrm{CO}\left(\mathrm{NH}_{2}\right)_{2}$<smiles>NC(N)=O</smiles>

Benzamide

$\mathrm{C}_{6} \mathrm{H}_{5} \mathrm{CONH}_{2}$<smiles>NC(=O)C1CCCCC1</smiles>

Ethylamine Hydrochloride

$\mathrm{C}_{2} \mathrm{H}_{5} \mathrm{NH}_{2} \cdot \mathrm{HCl}$<smiles>CCC(C)N</smiles>

Acetic Acid

$\mathrm{CH}_{3} \mathrm{COOH}$<smiles>CC(=O)O</smiles>

Sodium Acetate $\mathrm{CH}_{3}$ COONa<smiles>CC(=O)O[Na]</smiles> 
Figure 2
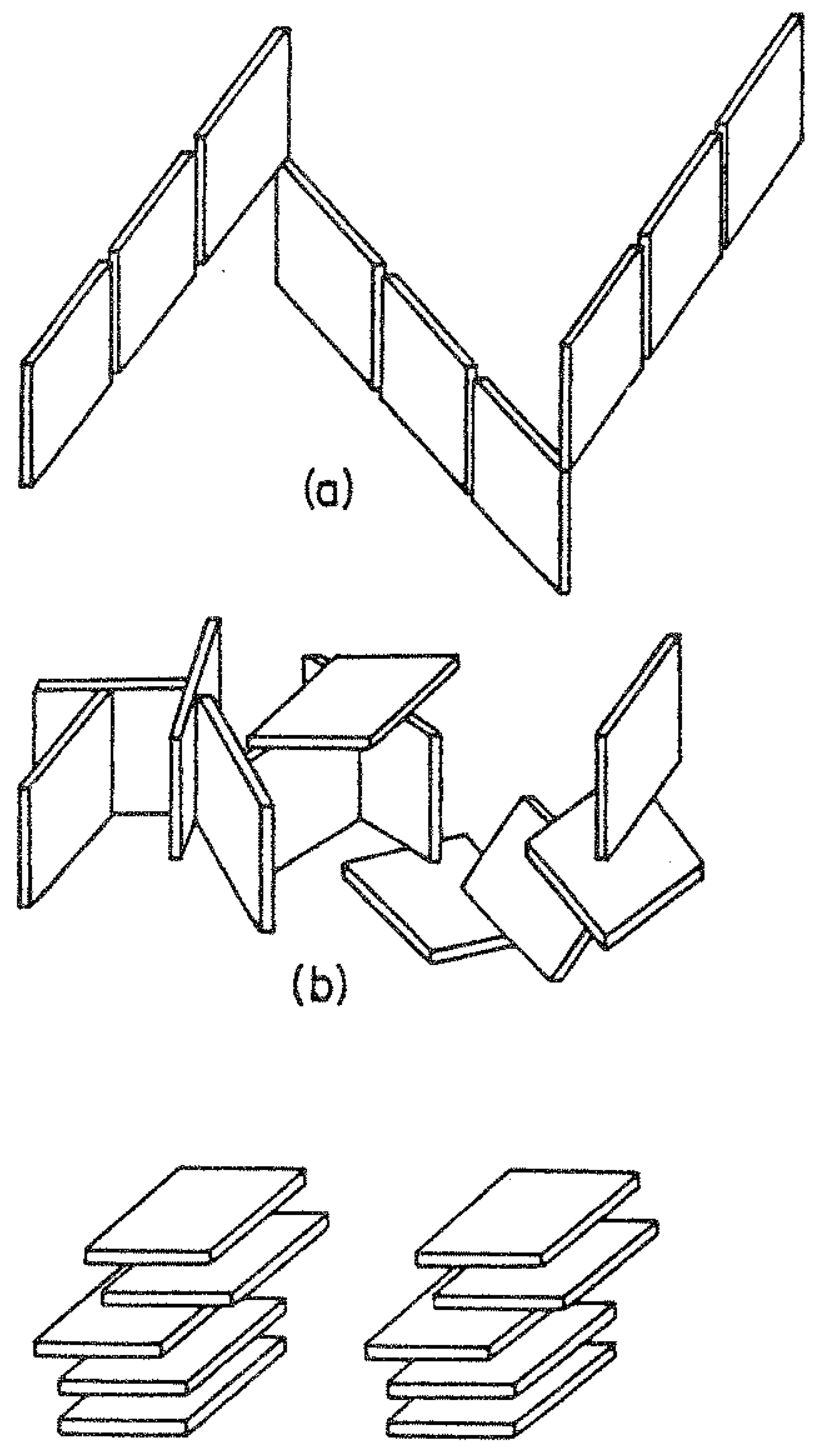

(c) 
Figure 3

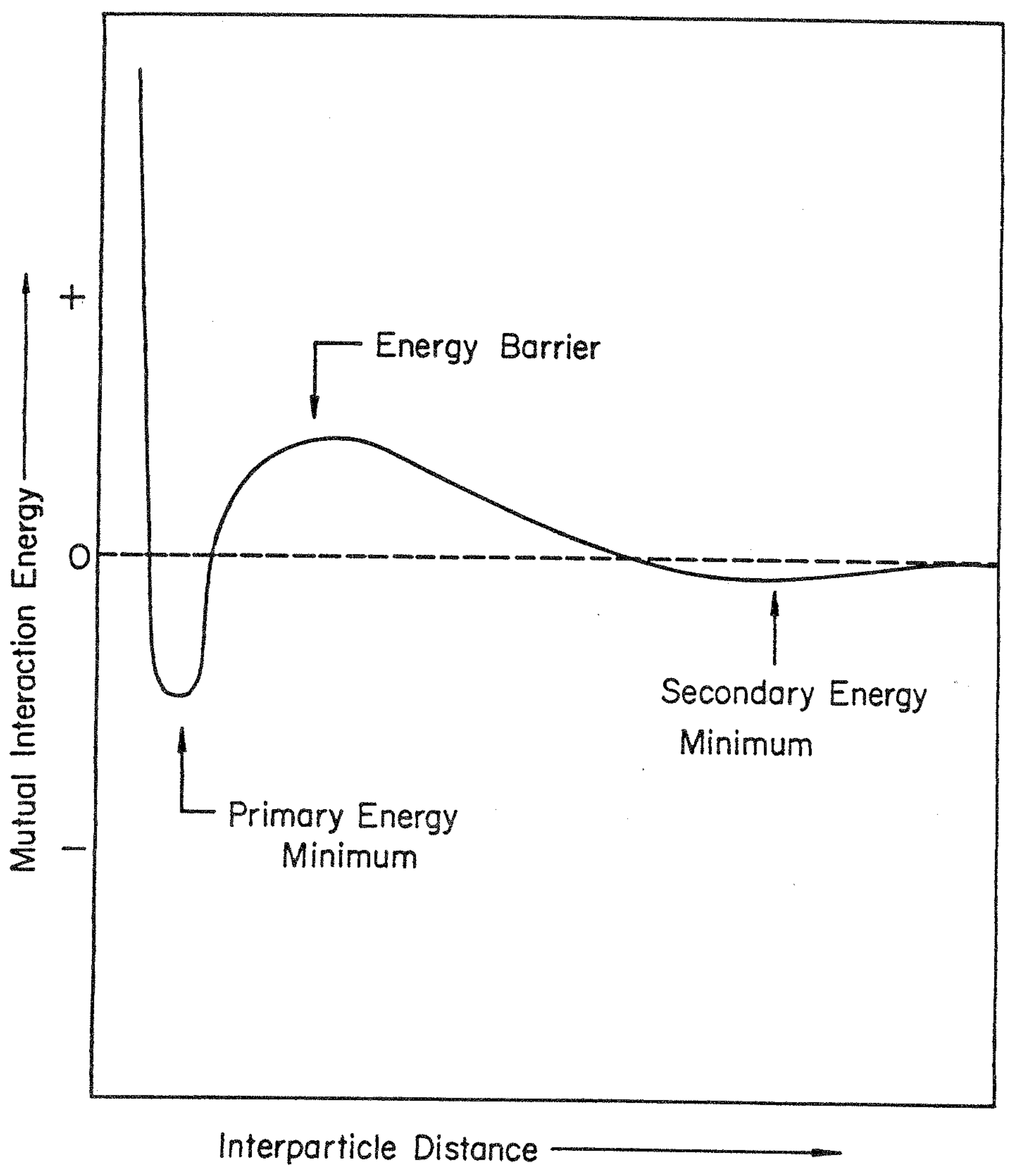


$m_{w} / m_{c}$

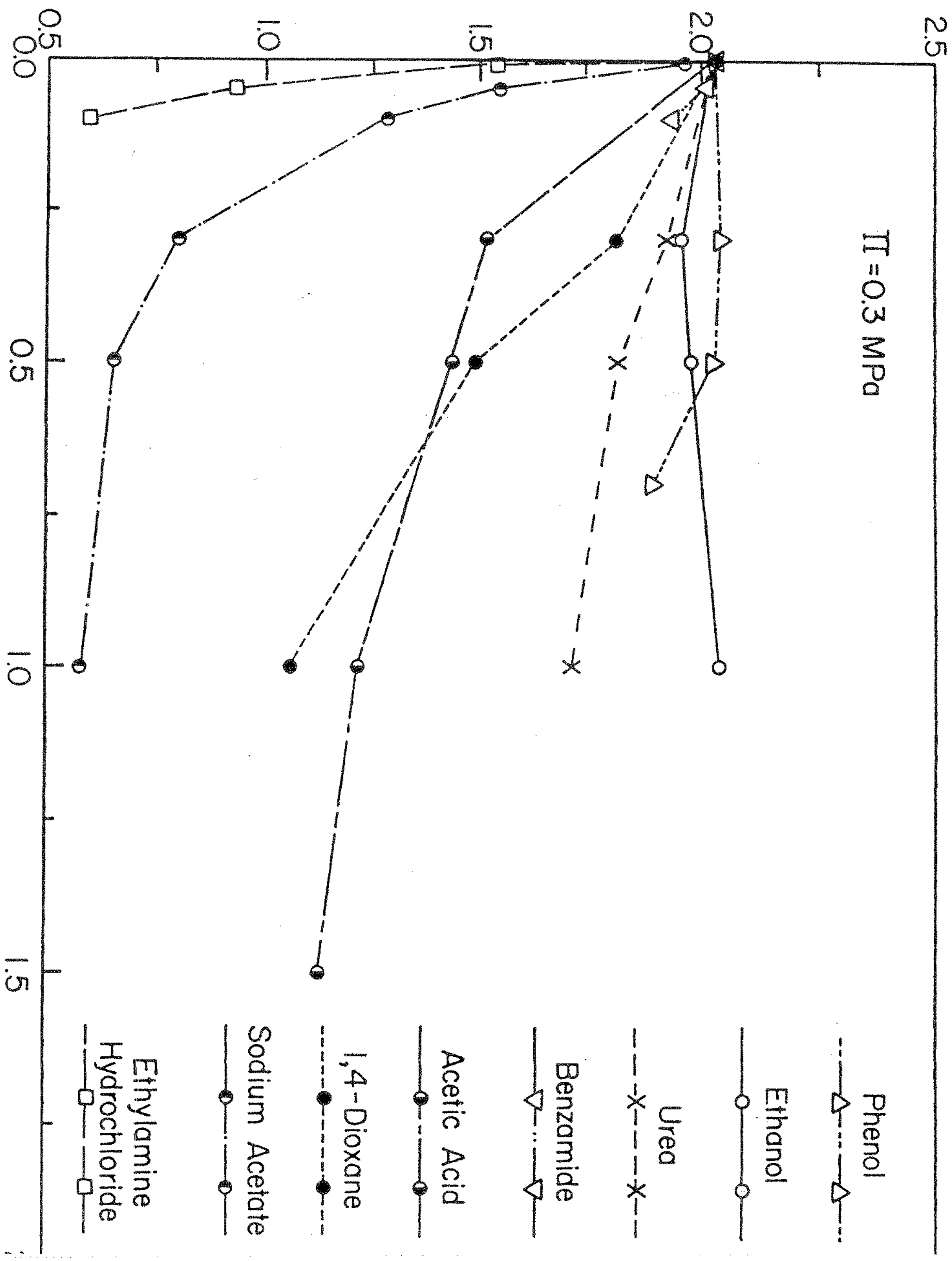


Figure 5

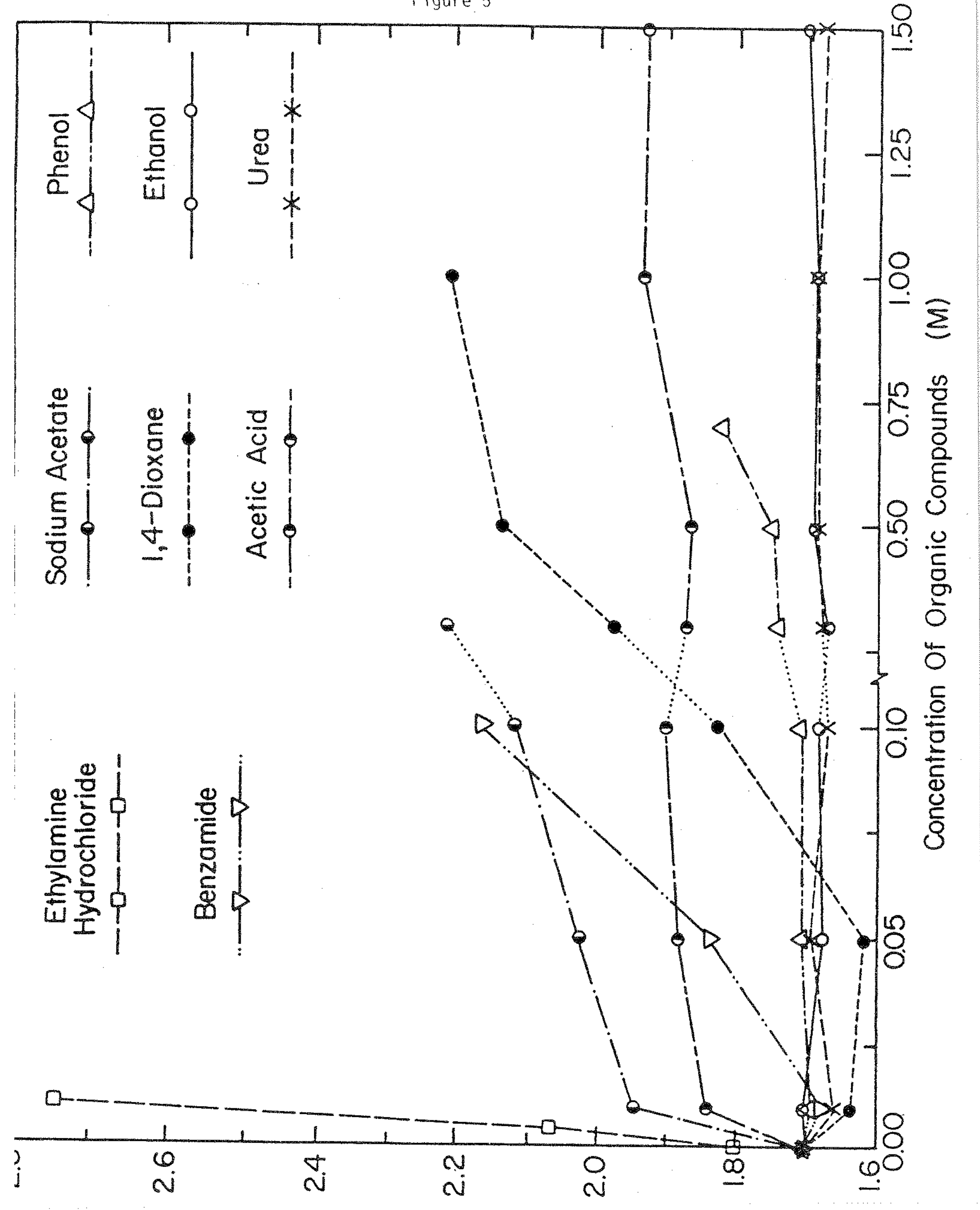




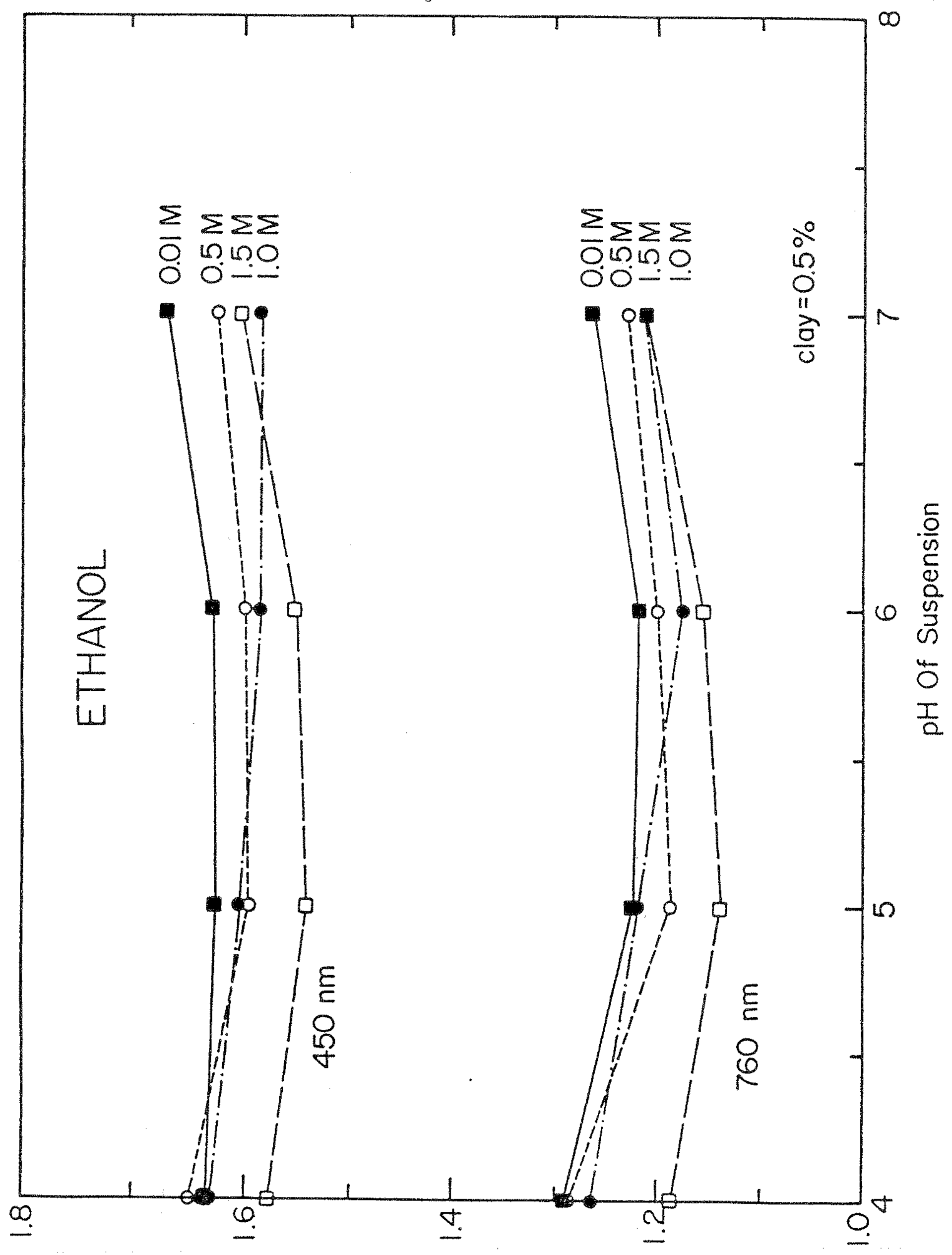




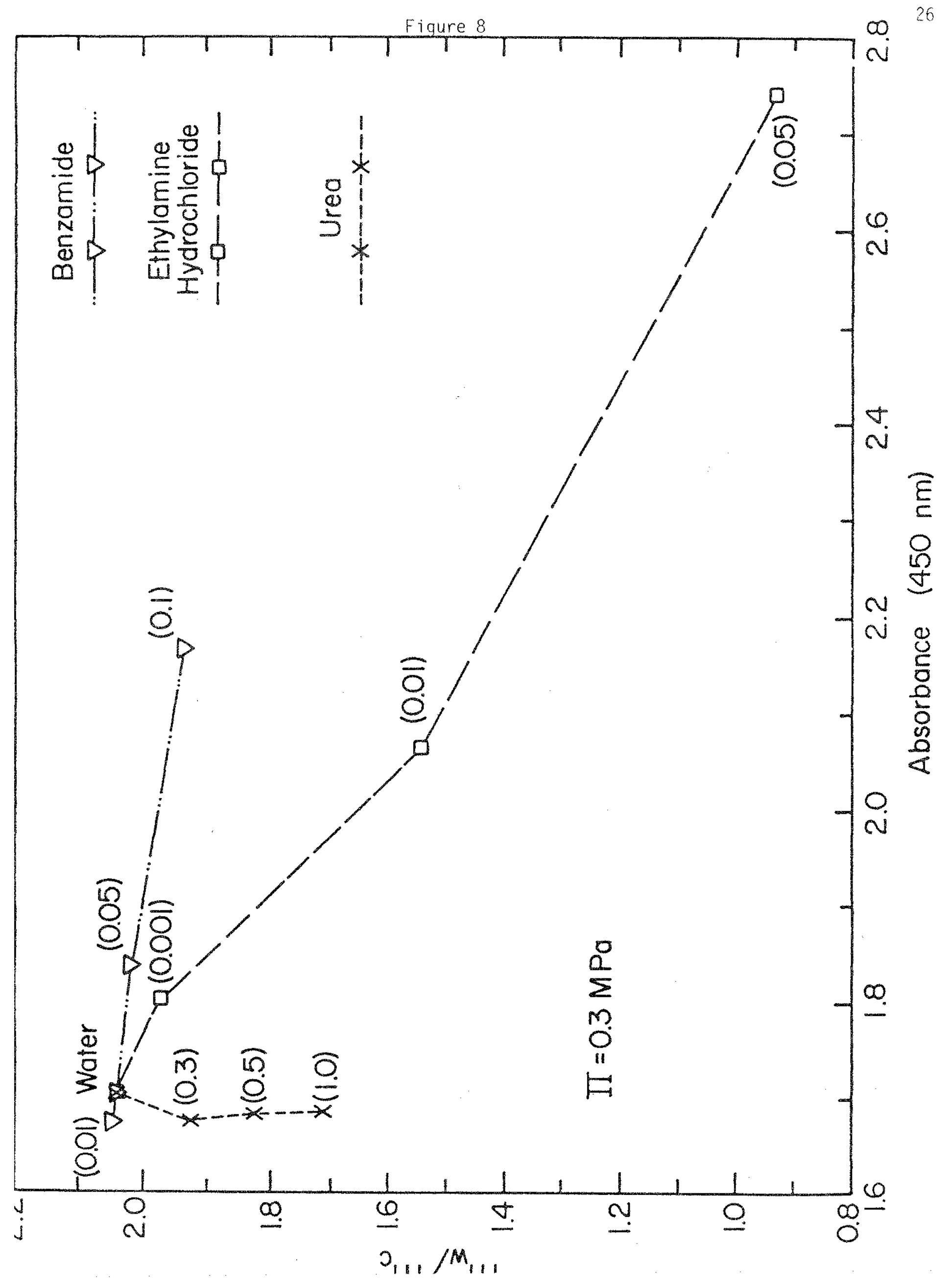




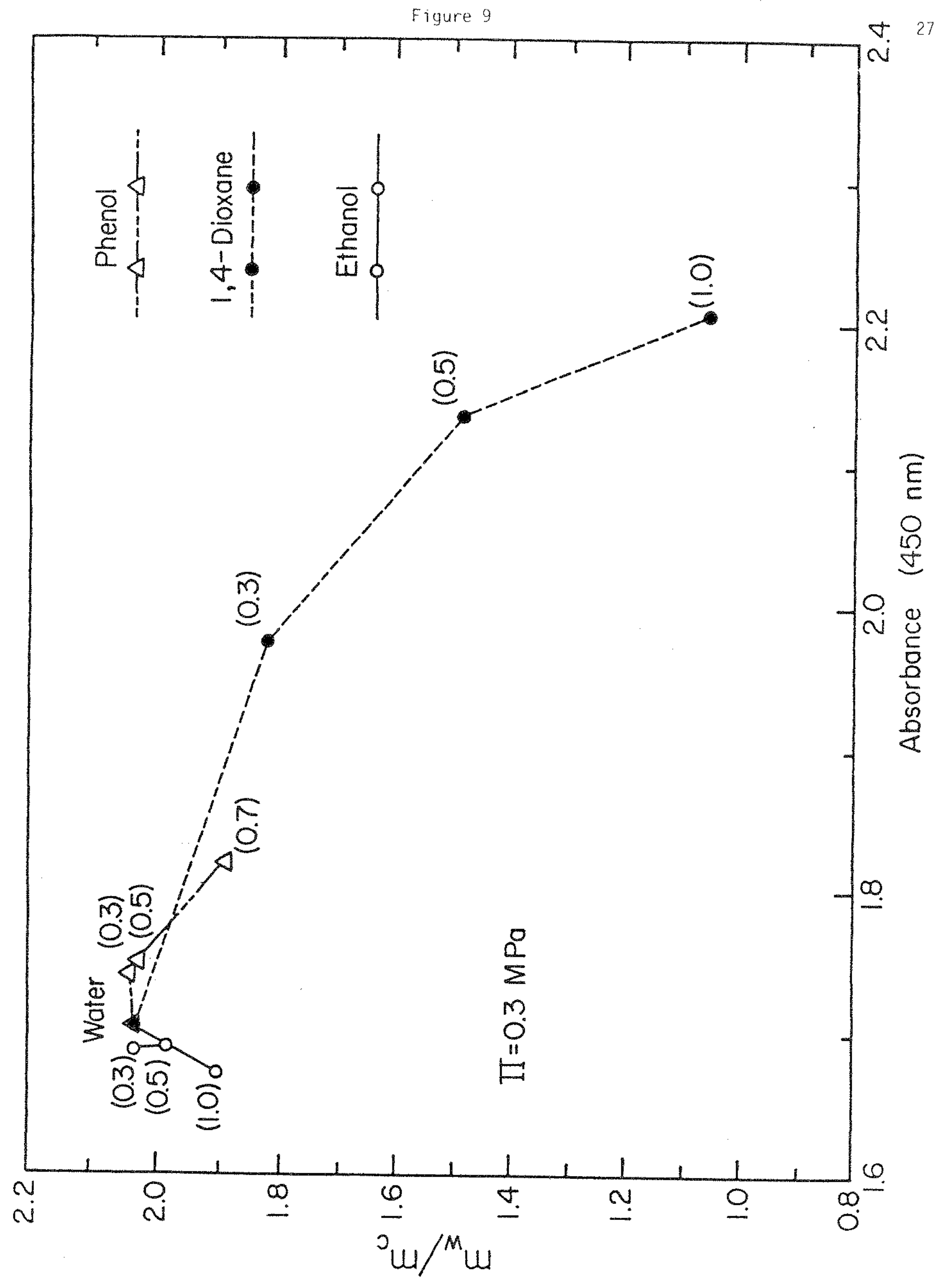


Figure 10

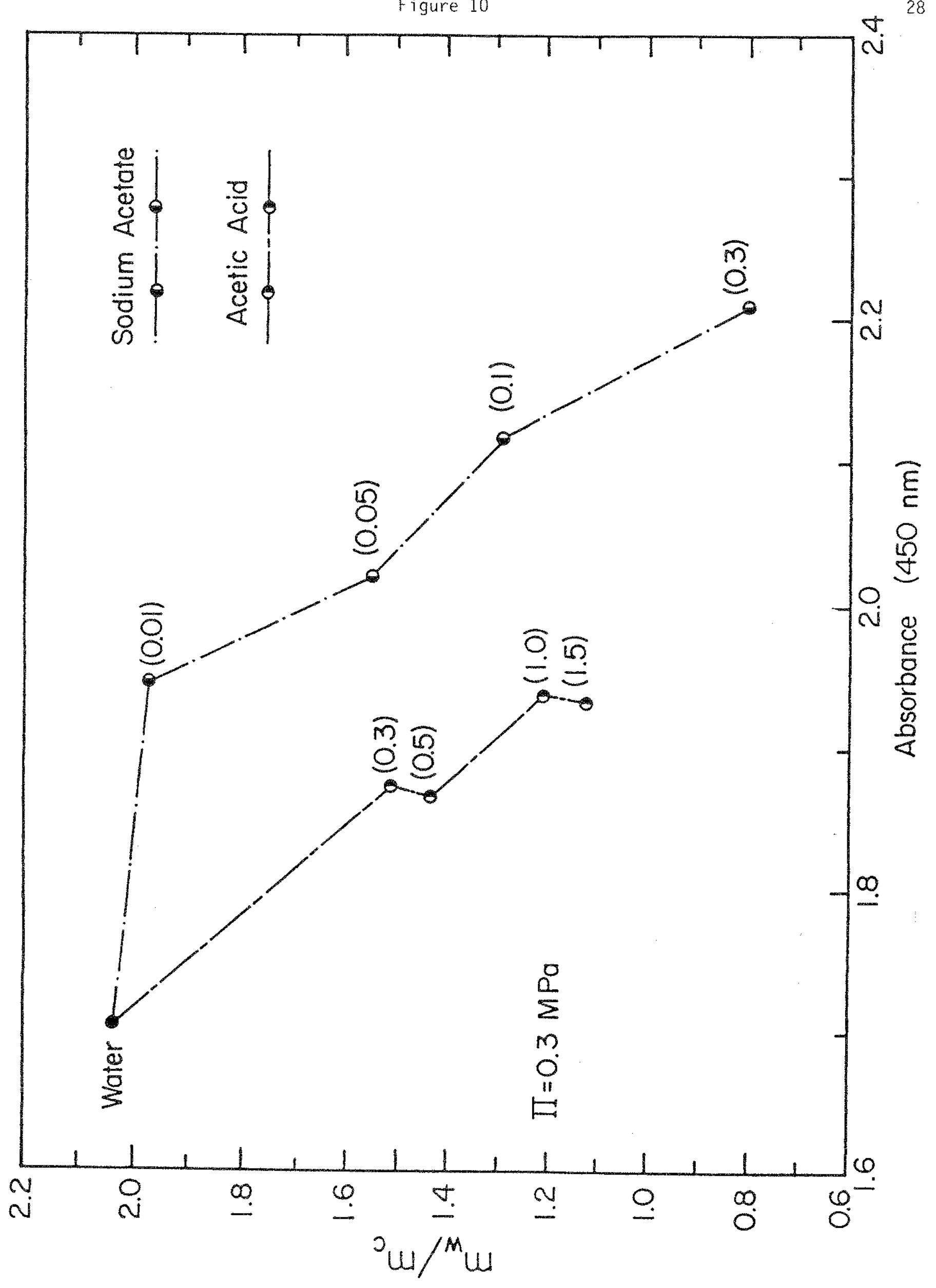

